\title{
ON THE DETECTION OF SUBPHOTOSPHERIC CONVECTIVE VELOCITIES AND TEMPERATURE FLUCTUATIONS*
}

\author{
D. O. GOUGH \\ Institute of Astronomy, and Department of Applied Mathematics and Theoretical Physics, University of \\ Cambridge \\ and \\ J. TOOMRE \\ Joint Institute for Laboratory Astrophysics, and Department of Astrophysical, Planetary and Atmospheric \\ Sciences, University of Colorado, Boulder, Colo., U.S.A.
}

\begin{abstract}
A procedure is outlined for estimating the influence of large-scale convective eddies on the wave patterns of five-minute oscillations of high degree. The method is applied to adiabatic oscillations, with frequency $\omega$ and wave number $k$, of a plane-parallel polytropic layer upon which is imposed a low-amplitude convective flow. The distortion to the $k-\omega$ relation has two constituents: one depends on the horizontal component of the convective velocity and has a sign which depends on the sign of $\omega / k$; the other depends on temperature fluctuations and is independent of the sign of $\omega / k$. The magnitude of the distortion is just at the limit of present observational sensitivity. Thus there is reasonable hope that it will be possible to reveal some aspects of the large-scale flow in the solar convection zone.
\end{abstract}

\section{Introduction}

The structure of the waves that produce the five-minute oscillations of the Sun depend principally on the mean vertical stratification of temperature. That stratification determines a fairly well defined sequence of relations between the frequency and the horizontal wave number of the oscillations. This has been exhibited for the real Sun in power spectra of Doppler measurements (e.g., Deubner et al., 1979), and compared with theory to determine the adiabat in the isentropic part of the convection zone (e.g., Berthomieu et al., 1980; Lubow et al., 1980). However, these relations are not perfectly maintained: frequency is not precisely determined because the modes do not persist indefinitely, and the wave patterns are distorted by rotation and the inhomogeneities associated with convection. It is the purpose of this paper to report a preliminary theoretical assessment of the magnitude of the distortions, with a view to the eventual measurement of the velocity and temperature fluctuations in the convection zone. In an accompanying paper (Hill et al., 1983) observational evidence for such distortions is presented.

We restrict our attention to modes of high degree. In that case the oscillations are trapped in a shallow wave guide just beneath the photosphere: the base of the trapping region is at a depth of about $n l^{-1} R$, where $n$ and $l$ are the order and degree of the mode

* Proceedings of the 66th IAU Colloquium: Problems in Solar and Stellar Oscillations, held at the Crimean Astrophysical Observatory, U.S.S.R., 1-5 September, 1981.

Solar Physics 82 (1983) 401-410. 0038-0938/83/0822-0401\$01.50.

Copyright (C) 1983 by D. Reidel Publishing Co., Dordrecht. Holland. and Boston, U.S.A. 
and $R$ is the radius of the Sun. Because the wave guide is shallow we may neglect the curvature of the Sun and consider the dynamics of a plane atmosphere under a constant gravitational acceleration $g$. Moreover, we may associate with the mode a constant horizontal wave number $k \simeq l R^{-1}$.

The solar envelope is considered to be in a state of convection. Convective fluctuations in the thermodynamic properties of the envelope are regarded as being small compared with the corresponding horizontally averaged values, so that linearization about the mean state is valid. Furthermore, we consider the influence on the waves of only the largest scales of convective motion - the giant cells, perhaps - so that the temporal and horizontal spatial variations of the convective flow can be ignored compared with those of the waves. Essentially we are retaining just the leading terms in a JWKB expansion in time and horizontal Cartesian co-ordinates. Thus, we compute locally the perturbation to the dispersion relation between the frequency $\omega$ and the horizontal wave number $k$.

We impose no restriction on the vertical scale of variation. Convection has a tendency to form thin horizontal boundary layers, and we wish to allow for the possibility that these may be no thicker than the vertical scale of the waves. Thus we have in mind, for example, the problem of determining whether there are shear layers in the giant cells, such as have been found theoretically by Latour et al. (1983) with the single-mode representation of convection.

\section{Perturbations to the Dispersion Relation}

We first ignore the large-scale convective motion. The stratification of the envelope can then be specified by any two thermodynamic state variables, which are functions of just the vertical co-ordinate $z$. Here we choose the adiabatic sound speed $c(z)$ and the adiabatic exponent $\gamma(z) \equiv(\partial \ln p / \partial \ln \rho)_{s}$, where $p$ and $\rho$ are pressure and density and the derivative is taken at constant specific entropy $s$. Any linear normal mode of oscillation can be represented as a superposition of waves with the same horizontal wave number $k$. We assume the wave motion to be adiabatic. A wave variable $\chi$, say, associated with such a wave can then be written in the separated form

$$
\chi(\mathbf{x}, t)=\operatorname{Re}\left[X(z) e^{i k x+i \omega t}\right]
$$

with respect to suitably orientated Cartesian co-ordinates $\mathbf{x} \equiv(x, y, z)$ and time $t$; the frequency $\omega$ is real (which, without loss of generality, we take to be positive) and depends on $|k|$ and the structure of the unperturbed atmosphere. It may be written

$$
\omega^{2}=\int f\left(n,|k| ; c^{2}, \gamma\right) \mathrm{d} z .
$$

Note that $\omega$ is independent of the horizontal direction of propagation of the wave.

The effect of the rotation of the Sun is simply to translate the wave pattern horizontally. We assume that this has been accounted for, and concentrate on the convective eddies. These influence the wave via a combination of advection and a modification to the 
function $f$ resulting from variations in $c^{2}$ and $\gamma$. We ignore the influence of the vertical component of the convective velocity, for in the high wave number limit considered here we expect it to be much smaller than the other contributions to the distortion of the wave pattern.

It is evident that only the $x$ component of the velocity of advection of the wave pattern represented by $(2.1)$ has any significance. Let this velocity be $\widetilde{U}$. Then the frequency $\omega$ is simply augmented by $k \tilde{U}$. Clearly, if the horizontal component of the convective velocity is independent of $z$ throughout the region within which the wave is trapped, $\tilde{U}$ is simply the $x$ component of that velocity. If the convective velocity varies with $z$, then once again $\tilde{U}$ depends only on its $x$ component $U(z)$; it is simply an average of $U$ weighted with the kinetic energy density of the wave (cf. Gough, 1978):

$$
\tilde{U}=\int B(z) U(z) \mathrm{d} z
$$

where

$$
B(z) \equiv \frac{\rho \mathbf{u} \cdot \mathbf{u}}{\int \rho \mathbf{u} \cdot \mathbf{u} \mathrm{d} z}
$$

and is independent of the sign of $k ; \mathbf{u}(z)$ is the velocity amplitude of the wave and $\rho$ is the density of the unperturbed envelope. The integrals are presumed to be taken over the entire extent of the envelope. Since horizontal density variations induced by the convection are presumed to be small, $\rho$ may be replaced by the horizontally averaged density $\bar{\rho}$, which is a function of $z$ alone.

The changes to the dispersion relation arising from the slow horizontal variation in the thermodynamic state of the gas can be obtained simply by expanding $c^{2}$ and $\gamma$ about their horizontally averaged values. Setting

$$
\begin{aligned}
c^{2}(\mathbf{x}, t) & =\overline{c^{2}}(z)+\delta c^{2}(\mathbf{x}, t), \\
\gamma(\mathbf{x}, t) & =\bar{\gamma}(z)+\delta \gamma(\mathbf{x}, t),
\end{aligned}
$$

one obtains formally for the linearized change $\delta \omega^{2}$ in $\omega^{2}$ :

$$
\delta \omega^{2}=\int \overline{c^{2}} \frac{\delta f}{\delta c^{2}} \frac{\delta c^{2}}{\overline{c^{2}}} \mathrm{~d} z+\int \bar{\gamma} \frac{\delta f}{\delta \gamma} \frac{\delta \gamma}{\bar{\gamma}} \mathrm{d} z
$$

where $\delta f / \delta c^{2}$ and $\delta f / \delta \gamma$ are the variational derivatives of $f$ with respect to $c^{2}$ and $\gamma$.

The results can be combined to yield an expression for the total local variation $\Delta \omega$ in the frequency $\omega$ of the component of the wave pattern whose local horizontal wave number is $k$. This may be written in the form

$$
\frac{\Delta \omega}{\omega}=\frac{k}{\omega} \int z B U \mathrm{~d} \ln z+\int F \frac{\delta c^{2}}{\overline{c^{2}}} \mathrm{~d} \ln z+\int G \frac{\delta \gamma}{\bar{\gamma}} \mathrm{d} \ln z
$$


An explicit expression for the kernel $F$, in the case when $\gamma$ is constant, is derived in the Appendix.

Notice that since $f$ is independent of the sign of $k$, so are $F$ and $G$. Thus the second and third terms on the right-hand side of Equation (2.7) are independent of the wave direction, whereas the first term has opposite signs for waves propagating in the positive and negative $x$ directions. Hence, if local dispersion relations can be measured for waves propagating in opposite directions, the contributions to their variations induced by large-scale convection can be partially separated by computing sums and differences of those variations.

\section{A Simple Illustration}

We illustrate the result by considering an envelope of infinite depth whose horizontally averaged structure is that of a polytrope of index $m$. In addition we take $\gamma$ to be constant.

In a polytropic model the square of the sound speed increases linearly with depth:

$$
c^{2}=\frac{\gamma g z}{m+1}
$$

where $z$ is measured downwards from the top of the envelope. Adiabatic $p$ modes of order $n$ have a dispersion relation given by

$$
\omega^{2}=\frac{\gamma g|k|}{m+1}\left\{n+\frac{1}{2} m+\left[\left(n+\frac{1}{2} m\right)^{2}-\frac{m+1}{\gamma}\left(m-\frac{m+1}{\gamma}\right)\right]^{1 / 2}\right\}
$$

(e.g. Gough, 1978). They are trapped in a region that extends roughly to the depth $z_{t}$ at which $\omega^{2}=k^{2} c^{2}$, namely where $c^{2}$ is such that pure plane acoustic waves of frequency $\omega$ and horizontal wave number $k$, if they could exist, would travel horizontally. They are also evanescent near the surface, very roughly at depths less than $z_{c}$ at which $\omega$ is equal to Lamb's acoustical cutoff frequency $\gamma g / 2 c$ for an isothermal atmosphere. The depths $z_{c}$ and $z_{\ell}$ are essentially the turning points of a JWKB analysis, though they do not correspond precisely unless the variables are chosen judiciously.

It is evident from Equations (3.1) and (3.2) that $z_{t}$ is insensitive to the structure of the envelope, and approaches $n k^{-1}$ as $n$ increases. On the other hand, $z_{c}$ is roughly inversely proportional to the gradient of the sound speed, and also to $n$.

The velocity amplitude of the $f$ mode is proportional to $e^{-|k| z}$, and the frequency is $(g|k|)^{1 / 2}$.

Kernels $B$ and $F$ are illustrated in Figures 1 and 2 for the $f$ mode and the first five $p$ modes. In this simple example, the kernels $G$ are zero. As one might expect, the depths of the main contributions to the kernels increase with $n$, since $z_{t}$ increases with $n$. The velocity kernels are, of course, positive everywhere. For $p$ modes the kernels $F$ are positive throughout most of the region, as one would expect because increasing $c^{2}$ tends to decrease the acoustical propagation time across the wave guide, thus increasing the 
resonant frequency. That is not so in the evanescent region at the top of the layer, however, because there an increase in $c^{2}$ results in a decrease in the acoustical cutoff frequency. This increases the extent of the region within which the waves can propagate, and so increases the sound travel time across the trapping region. No such reversal in the sign of $F$ occurs at the bottom of the trapping region, since $z_{t}$ is intensitive to $c^{2}$. The kernel $F$ is identically zero for the $f$ modes, because the frequencies of $f$ modes in a plane parallel atmosphere are independent of stratification.

To model a possible giant cell, velocity and temperature fluctuations were obtained from a steady two-dimensional convective flow of a fluid with $\gamma=\frac{5}{3}$. The flow was computed by N. Hurlburt using the numerical programme described by Graham (1975). The Rayleigh number was $10^{6}$ and the Prandtl number was unity, and the mean polytropic index characterizing the horizontally averaged density stratification was about 1.2. The convecting region extended over about 3.6 density scale heights. Amplitudes of the vertical component of velocity and the temperature fluctuation, $W$ and $\delta T$, were estimated by taking at each value of $z$ half the difference between the values of velocity and temperature at the two sides of the cell, where the velocity was constrained to be vertical. The horizontal velocity amplitude $U$ was taken to be the horizontal component of velocity on the vertical line midway between the sides. These functions were used to represent the giant-cell flow in the bottom 3.6 scale heights of the convection zone. The relative temperature fluctuation $\delta T / T$ was used without modification and the velocity was scaled by a constant factor, chosen to make the maximum value of the rms vertical component equal to $50 \mathrm{~m} \mathrm{~s}^{-1}$; this is roughly consistent with mixing-length estimates at the height at which the maximum occurs. Beneath the convection zone, which is assumed to be $200 \mathrm{Mm}$ deep, convective fluctuations were presumed to vanish; above the region modelled by Hurlbert's solution the temperature fluctuation and the vertical velocity were set to zero, and the horizontal velocity was assumed to be constant and continuous with the flow beneath. The result is illustrated in Figure 3.

The contributions from $U$ and $\delta T / T=\delta c^{2} / \overline{c^{2}}$ to the frequency deviation were computed from the appropriate terms in Equation (2.7), using the kernels depicted in

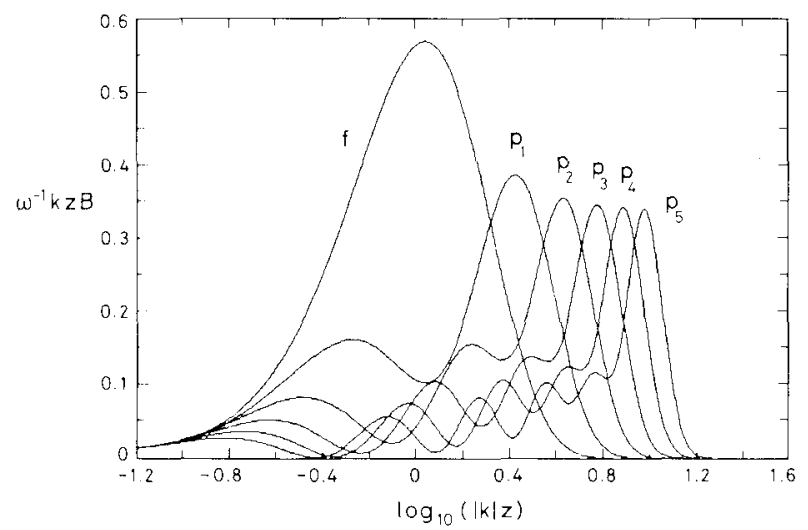

Fig. 1. Kernels $\omega^{-1} k z B$ for a polytrope of index $m=1.2$ with $\gamma=\frac{5}{3}$, measured in units of $(k / g)^{1 / 2}$. 


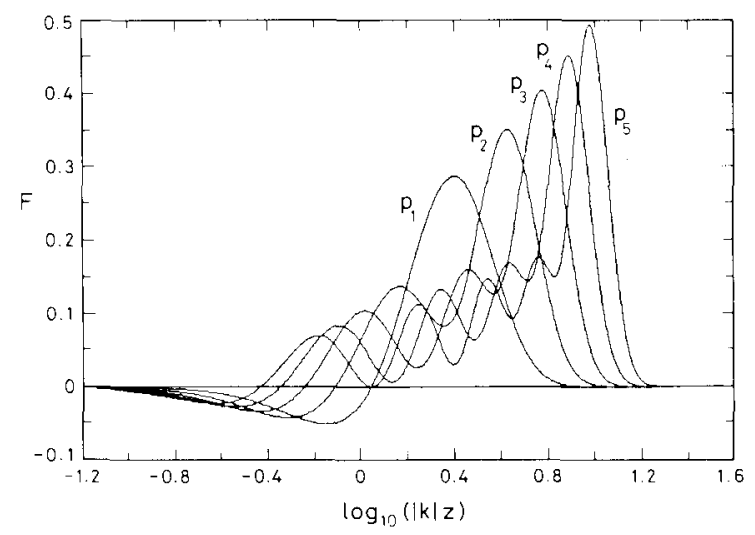

Fig. 2. Kernels $F$ for a polytrope of index $m=1.2$ with $\gamma=\frac{5}{3}$.

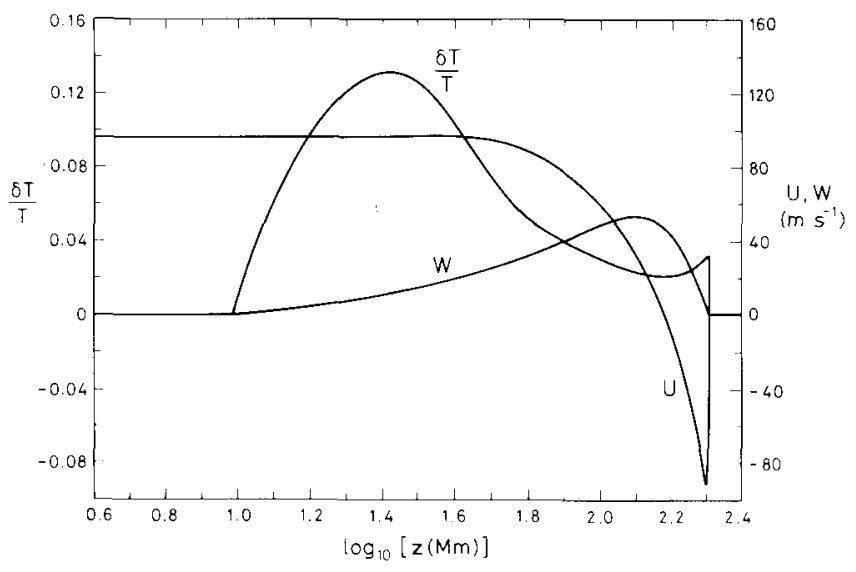

Fig. 3. Horizontal and vertical components $(U, W)$ of velocity and relative temperature fluctuation $\delta T / T$ associated with the assumed large-scale convective flow.

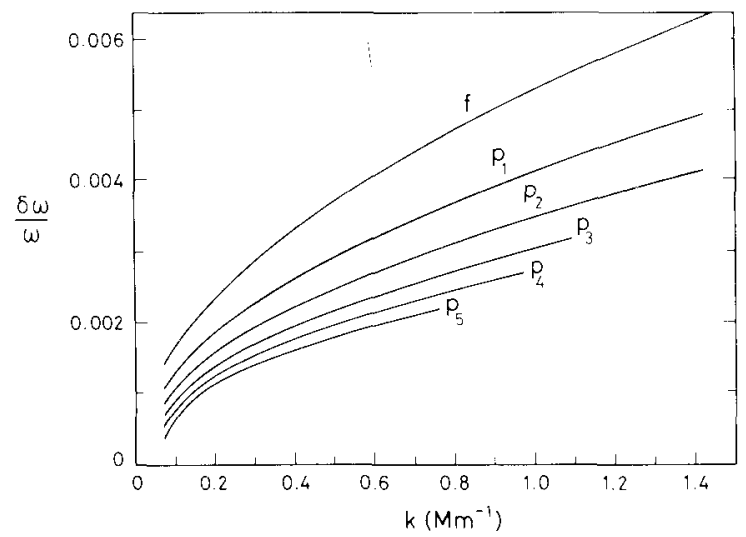

Fig. 4. Relative frequency perturbations for $k>0$ produced by the horizontal component of velocity illustrated in Figure 3. The perturbations for $-k$ have the same magnitude, but opposite sign. 
Figures 1 and 2. The results are shown in Figures 4 and 5. Notice that the contribution from the temperature fluctuation is such that it should be detectable by modern observations, for the half-widths of the bands in the power spectrum of Deubner et al. (1979) are only of order one per cent. The velocity contribution is also large enough to be interesting, especially since the modal computations of Latour et al. (1983) suggest that we may have underestimated the speed of the horizontal flow.

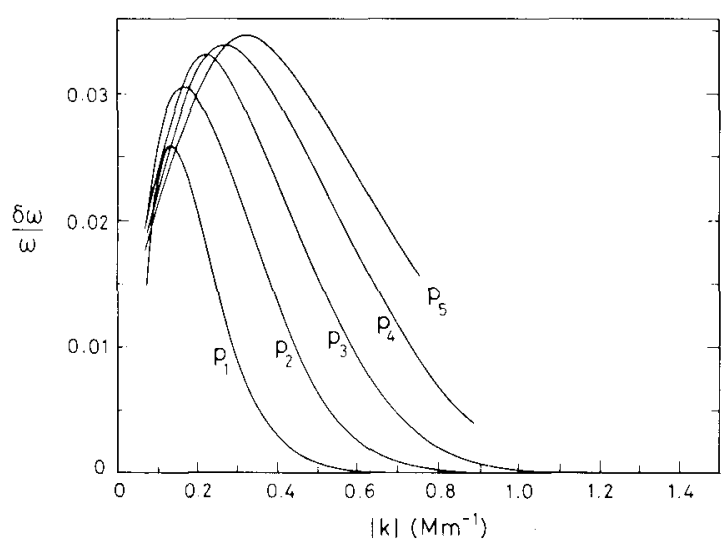

Fig. 5. Relative frequency perturbations produced by the temperature fluctuation illustrated in Figure 3. The perturbation is independent of the sign of $k$.

The forms of the frequency changes are also noteworthy. The modes are confined to a layer whose depth is about $n k^{-1}$, and only in that layer do they experience the mean structure of the envelope. Thus at high $k$ the velocity contribution to $\Delta \omega / \omega$ is simply that of advection by the surface value of $U$. But as $k$ decreases a more extensive average of $U$ is taken. Since $U$ decreases monotonically with depth, $\Delta \omega$ decreases with diminishing $k$, and at the lowest values of $k$ (not shown in Figure 4) the frequency perturbations change sign. The relative temperature fluctuation, on the other hand, has a maximum at a depth of about $25 \mathrm{Mm}$, and its contribution to $\Delta \omega$ is greatest when $k$ is such that the main weight of the kernel occurs also at this depth.

We have not attempted to estimate from these results how an actual power spectrum would be affected. It is evident that if the horizontal scale of giant cells is comparable with the region of the solar disk over which the observations are made, the convective fluctuations would both broaden and distort the bands of power. The distortions would vary with time as the cells are caused to drift across the field of view by the rotation of the Sun. Moreover, the different contributions to the distortion will be out of phase, the temperature contribution being a maximum roughly when the velocity contribution is zero. There is evidence for such behaviour in the observations of Hill et al. (1982), though the data are not sufficiently free from noise for us to be sure that they have been interpreted correctly. 


\section{Conclusion}

Large-scale subphotospheric convective motion in the Sun is likely to be of a magnitude just sufficient to be detected by present observations of five-minute oscillations of high degree. The form of the distortion to the $k-\omega$ power spectra depends on the structure of the flow in the convective eddy. With longer more and precise observations it should be possible to measure some aspects of that flow.

\section{Acknowledgements}

We are grateful to $\mathrm{Mr} \mathrm{N}$. Hurlburt for making his model of convection available to us. The work reported in this paper was carried out whilst J.T. held a Science Research Council Senior Visiting Fellowship at the Department of Applied Mathematics and Theoretical Physics in Cambridge, and subsequently when D.O.G. was a visitor at the Joint Institute for Laboratory Astrophysics, which is operated jointly by the University of Colorado and the National Bureau of Standards. The research was also supported in part by the National Aeronautics and Space Administration by grants NSG-7511 and NAGW-91, and by the US Air Force Geophysics Laboratory by contract F196228-77C-0104.

\section{Appendix: $F$ : the $c^{2}$ Kernel}

We restrict attention to constant $\gamma$, and start from Lamb's (1932) form of the equation describing adiabatic waves in a plane parallel atmosphere:

$$
\begin{aligned}
& \left(\frac{\mathrm{d}^{2}}{\mathrm{~d} z^{2}}-k^{2}\right) X+\left(\frac{\mathrm{d} \ln c^{2}}{\mathrm{~d} z}+\frac{\gamma g}{c^{2}}\right) \frac{\mathrm{d} X}{\mathrm{~d} z}+ \\
& +\left\{\frac{\omega^{2}}{c^{2}}-\frac{g k^{2}}{\omega^{2}}\left[\frac{\mathrm{d} \ln c^{2}}{\mathrm{~d} z}-(\gamma-1) \frac{g}{c^{2}}\right]\right\} X=0,
\end{aligned}
$$

where $X(z)$ is the (real) amplitude of $\chi=\operatorname{div} \mathbf{u}$, as in Equation (2.1), and $z$ is measured downwards. We shall assume that $c^{2}=0$ at $z=0$, and choose solutions $X$ that are regular at $z=0$ and that tend to zero as $z \rightarrow \infty$.

The equation may be cast into self-adjoint form by multiplying it by the factor $c^{2} \psi$, where

$$
\psi=\exp \left[-\gamma g \int_{z}^{Z} c^{-2}(s) \mathrm{d} s\right]
$$

and $Z$ is any positive constant. Multiplying by $X$ and integrating then yields

$$
\omega^{4} \int_{0}^{\infty} X^{2} \psi \mathrm{d} z-\omega^{2} \int_{0}^{\infty} c^{2}\left(X^{\prime 2}+k^{2} X^{2}\right) \psi \mathrm{d} z-\int_{0}^{\infty} \Omega^{4} X^{2} \psi \mathrm{d} z=0,
$$


where

$$
\Omega^{4}=g k^{2}\left[\frac{\mathrm{d} c^{2}}{\mathrm{~d} z}-(\gamma-1) g\right]
$$

and a prime denotes differentiation with respect to the argument. The equation provides a variational principle for the problem: the stationary values of $\omega^{2}(X)$ defined by Equation (A3) amongst all functions $X$ that satisfy the boundary conditions are the eigenvalues of Equation (A1), and occur when $X$ is an eigenfunction.

It is now a simple matter to compute from Equation (A3) the linearized change $\delta \omega^{2}$ in $\omega^{2}$ that occurs when $c^{2}$ is changed to $c^{2}+\delta c^{2}$. In view of the stationary property of (A3), variations in $X$ that arise from perturbing the equilibrium state do not affect $\omega^{2}$ to leading order, and therefore need not be calculated. The linearized perturbation to Equation (A3) is thus

$$
\begin{aligned}
& \gamma g \int_{0}^{\infty}\left[\left(\omega^{4}-\Omega^{4}\right) X^{2}-\omega^{2} c^{2}\left(X^{\prime 2}+k^{2} X^{2}\right)\right] \psi \mathrm{d} z \int_{z}^{z} c^{-4}(s) \delta c^{2}(s) \mathrm{d} s- \\
& -\omega^{2} \int_{0}^{\infty}\left(X^{\prime 2}+k^{2} X^{2}\right) \psi \delta c^{2} \mathrm{~d} z+g k^{2} \int_{0}^{\infty}\left(X^{2} \psi\right)^{\prime} \delta c^{2} \mathrm{~d} z-I \delta \omega^{2}=0
\end{aligned}
$$

where

$$
I=\int_{0}^{\infty}\left[c^{2}\left(X^{\prime 2}+k^{2} X^{2}\right)-2 \omega^{2} X^{2}\right] \psi \mathrm{d} z
$$

After interchanging the order of integration in the double integral, and taking the limit $Z \rightarrow \infty$, Equation (A5) may be rewritten

$$
\frac{\delta \omega^{2}}{\omega^{2}}=2 \int_{0}^{\infty} F \frac{\delta c^{2}}{c^{2}} \mathrm{~d} \ln z
$$

where

$$
\begin{aligned}
F= & \frac{1}{2} z \omega^{-2} I^{-1}\left\{\gamma g c^{-2} \int_{0}^{z}\left[\left(\omega^{4}-\Omega^{4}\right) X^{2}-\omega^{2} c^{2}\left(X^{\prime 2}+k^{2} X^{2}\right)\right] \psi \mathrm{d} s-\right. \\
& \left.-\left[\omega^{2} c^{2}\left(X^{\prime 2}+k^{2} X^{2}\right)-g k^{2}\left(\gamma g X+2 c^{2} X^{\prime}\right) X\right] \psi\right\} .
\end{aligned}
$$

In the case of the polytrope of index $m, c^{2}$ is given by Equation (3.1), $\psi$ is proportional to $z^{m+1}, \quad \Omega^{4}=g^{2} k^{2}(m+1-m \gamma) /(m+1), \quad \omega^{2}$ is given by Equation (3.2) and $X=e^{-|k| z} L_{n}^{(m+2)}(2|k| z)$, where $L$ is a Laguerre polynominal. 


\section{References}

Berthomieu, G., Cooper, A. J., Gough, D. O., Osaki, Y., Provost, J., and Rocca, A.: 1980, in H. A. Hill and W. A. Dziembowski (eds.), Nonradial and Nonlinear Stellar Pulsation, Springer, Heidelberg, p. 307.

Deubner, F.-L., Ulrich, R. K., and Rhodes, Jr. E. J.: 1979, Astron. Astrophys. 72, 177.

Gough, D. O.: 1978, in G. Belvedere and L. Paternò (eds.), Proc. Workshop on Solar Rotation, University of Catania Press, Catania, p. 255.

Graham, E.: 1975, J. Fluid Mech. 70, 689.

Hill, F., Toomre, J., and November, L.: 1983, Solar Phys. 82, 411 (this volume).

Lamb, H.: 1932, Hydrodynamics, Cambridge University Press, Cambridge.

Latour, J., Toomre, J., and Zahn, J.-P.: 1983, Solar Phys. 82, 387 (this volume).

Lubow, S. H., Rhodes, Jr. E. J., and Ulrich, R. K.: 1980, in H. A. Hill and W. A. Dziembowski (eds.), Nonradial and Nonlinear Stellar Pulsation, Springer, Heidelberg, p. 300. 